\title{
Behavior of a Scale Factor for Wiener Integrals and a Fourier Stieltjes Transform on the Wiener Space
}

\author{
Young Sik Kim \\ Department of Mathematics, College of Natural Sciences, Hanyang University, Seoul, South Korea \\ Email: yoskim@hanyang.ac.kr
}

How to cite this paper: Kim, Y.S. (2018) Behavior of a Scale Factor for Wiener Integrals and a Fourier Stieltjes Transform on the Wiener Space. Applied Mathematics, 9, 488-495.

https://doi.org/10.4236/am.2018.95035

Received: March 16, 2018

Accepted: May 25, 2018

Published: May 28, 2018

Copyright $\odot 2018$ by author and Scientific Research Publishing Inc. This work is licensed under the Creative Commons Attribution International License (CC BY 4.0).

http://creativecommons.org/licenses/by/4.0/

\begin{abstract}
The purpose of this paper is to investigate the behavior of a Wiener integral along the curve $C$ of the scale factor $\rho>0$ for the Wiener integral $\int_{C_{0}[0, T]} F(\rho x) \mathrm{d} m(x)$ about the function $F(x)=\exp \left\{\int_{0}^{T} \theta(t, x(t)) \mathrm{d} t\right\}$ defined on the Wiener space $C_{0}[0, T]$, where $\theta(t, u)$ is a Fourier-Stieltjes transform of a complex Borel measure.

\section{Keywords}

Wiener Space, Wiener Integral, Feynman Integral, Analytic Wiener Integral, Analytic Feynman Integral, Fourier-Stieltjes Transform, Change of Scale Formula, Scale Factor
\end{abstract}

\section{Introduction}

In [1], M. D. Brue introduced the functional transform on the Feynman integral (1972). In [2], R. H. Cameron wrote the paper about the translation pathology of a Wiener space (1954). In [3] [4] [5], R. H. Cameron and W. T. Martin proved some theorems on the transformation and the translation and used the expression of the change of scale for Wiener integrals (1944-1947). In [6] and [7], R. H. Cameron and D. A. Storvick, proved relationships between Wiener integrals and analytic Feynman integrals to prove a change of scale formula for Wiener integrals (1987). In [8] and [9], properties among the schrödinger operator and the Wiener Integral and the Feynman integral and the Feynman's operational calculus were studied. In [10], G. W. Johnson and D. L. Skoug proved a scale-invariant measurability on the Wiener space (1979). 
In [11] and [12], Y. S. Kim proved relationships between Wiener integrals and analytic Feynman integrals and proved a change of scale formula for Wiener integrals about cylinder functions on the abstract Wiener space (1998-2001). In [13] [14] [15] [16], Kim proved relationships among the Fourier transform and the Fourier Feynman transform and the convolution on the abstract Wiener space (2006-2016).

In this paper, we define the scale factor for the Wiener integral and we investigate the behavior of Wiener integrals along the curve $C$ of a scale factor $\rho>0$ about complex valued measurable functions $F(x)=\exp \left\{\int_{0}^{T} \theta(t, x(t)) \mathrm{d} t\right\}$ defined on the Wiener space $C_{0}[0, T]$, where $\theta(t, u)=\int_{R} \exp \{i u v\} \mathrm{d} \sigma_{t}(v)$ is a Fourier-Stieltjes transform of a complex Borel measure $\sigma_{t}$. And we will find a very interesting behavior of a scale factor $\rho>0$ for the Wiener integral.

\section{Definitions and Preliminaries}

A collection $\mathcal{S}$ of subsets of a set $X$ is said to be a $\sigma$-algebra in $X$ if $\mathcal{M}$ has the following properties: 1) $X \in \mathcal{M}, 2$ ) If $A \in \mathcal{M}$, then $A^{t} \in \mathcal{M}$, (where $A^{t}$ is the complement of $A$ relative $X), 3$ ) If $A \in \bigcup_{n=1}^{\infty} A_{n}$ and $A_{n} \in \mathcal{S}$ for $n=1,2,3, \cdots$, then $A \in \mathcal{S}$.If $\mathcal{S}$ is a $\sigma$-algebra in $X$, then $X$ is called a measurable space and the members of $\mathcal{S}$ are called the measurable set in $X$. If $X$ is a measurable space and $Y$ is a topological space and $f$ is a mapping of $X$ into $Y$, then $f$ is a Lebesgue-measurable function, or more briefly, a measurable function, provided that $f^{-1}(V)$ is a measurable set in $X$ for every open set $V$ in $Y$.

Let $C_{0}[0, T]$ denote the space of real-valued continuous functions $\boldsymbol{X}$ on $[0, T]$ such that $x(0)=0$. Let $\mathcal{M}$ denote the class of all Wiener measurable subsets of $C_{0}[0, T]$ and let $m$ denote Wiener measure and $\left(C_{0}[0, T], \mathcal{M}, m\right)$ be a Wiener measure space and we denote the Wiener integral of a functional $F$ by $\int_{C_{0}[0, T]} F(x) \mathrm{d} m(x)$. A subset $E$ of $C_{0}[0, T]$ is said to be scale-invariant measurable if $\rho E \in \mathcal{M}$ for each $\rho>0$, and a scale-invariant measurable set $N$ is said to be scale-invariant null if $m(\rho N)=0$ for each $\rho>0$. A property that holds except on a scale-invariant null set is said to hold scale-invariant almost everywhere (s-a.e.). If two functionals $F$ and $G$ are equal s-a.e., we write $F \approx G$ (for more details, see [9]).

Throughout this paper, let $\mathbf{R}^{n}$ denote the $n$-dimensional Euclidean space and let $\mathbf{C}, \mathbf{C}_{+}$, and $\mathbf{C}_{+}^{\sim}$ denote the complex numbers, the complex numbers with positive real part, and the non-zero complex numbers with nonnegative real part, respectively.

Definition 2.1. Let $F$ be a complex-valued measurable function on $C_{0}[0, T]$ such that the integral

$$
J(F ; \lambda)=\int_{C_{0}[0, T]} F\left(\lambda^{-\frac{1}{2}} x\right) \mathrm{d} m(x)
$$

exists for all real $\lambda>0$. If there exists a function $J^{*}(F ; z)$ analytic on $\mathbf{C}_{+}$ such that $J^{*}(F ; \lambda)=J(F ; \lambda)$ for all real $\lambda>0$, then we define $J^{*}(F ; z)$ to 
be the analytic Wiener integral of $F$ over $C_{0}[0, T]$ with parameter $z$, and for each $z \in \mathbf{C}_{+}$, we write

$$
I^{a w}(F ; z)=J^{*}(F ; z) .
$$

Let $q$ be a non-zero real number and let $F$ be a function on $C_{0}[0, T]$ whose analytic Wiener integral exists for each $z$ in $\mathbf{C}_{+}$. If the following limit exists, then we call it the analytic Feynman integral of $F$ over $C_{0}[0, T]$ with parameter $q$, and we write

$$
I^{a f}(F ; q)=\lim _{z \rightarrow-i q} I^{a w}(F ; z),
$$

where $z$ approaches $-i q$ through $\mathbf{C}_{+}$and $i^{2}=-1$

Now we introduce the following Wiener Integration Formula.

Theorem 2.2. Let $C_{0}[0, T]$ be a Wiener space and let $0 \leq t_{1} \leq t_{2} \leq \cdots \leq t_{n} \leq T$. Then

$$
\begin{aligned}
& \int_{C_{0}[0, T]} f\left(x\left(t_{1}\right), x\left(t_{2}\right), \cdots, x\left(t_{n}\right)\right) \mathrm{d} m(x) \\
& =\left[\prod_{j=1}^{n} 2 \pi\left(t_{j}-t_{j-1}\right)\right]^{-\frac{1}{2}} \cdot \int_{\mathbf{R}^{n}} f(\vec{u}) \exp \left\{-\frac{1}{2} \sum_{j=1}^{n} \frac{\left(u_{j}-u_{j-1}\right)^{2}}{t_{j}-t_{j-1}}\right\} \mathrm{d} \vec{u},
\end{aligned}
$$

where $f: \mathbf{R}^{n} \rightarrow \mathbf{C}$ is a Lebesgue measurable function and $\vec{u}=\left(u_{1}, u_{2}, \cdots, u_{n}\right)$ and $\mathrm{d} \vec{u}=\mathrm{d} u_{1} \mathrm{~d} u_{2} \cdots \mathrm{d} u_{n}$.

In the next section, we will use the following integration formula:

$$
\int_{\mathbf{R}} \exp \left\{-a u^{2}+i b u\right\} \mathrm{d} u=\sqrt{\frac{\pi}{a}} \exp \left\{-\frac{b^{2}}{4 a}\right\},
$$

where $a$ is a complex number with $\operatorname{Re} a>0, b$ is a real number, and $i^{2}=-1$.

\section{Behavior of a Scale Factor for the Wiener Integral}

We investigate the behavior of the scale factor for the function space integral for functions

$$
F(x)=\exp \left\{\int_{0}^{T} \theta(t, x(t)) \mathrm{d} t\right\} .
$$

Definition 3.1. Let $\theta:[0, T] \times R \rightarrow C$ be defined by

$$
\theta(t, u)=\int_{R} \exp \{i u v\} \mathrm{d} \sigma_{t}(v),
$$

which is a Fourier-Stieltjes transform of a complex Borel measure $\sigma_{t} \in \mathbf{M}(R)$ with $\left\|\sigma_{t}\right\|<\infty$, where $\mathbf{M}(R)$ is a set of complex Borel measures defined on $R . \square$

Remark. If we define a function on $R$ by $f(u)=\theta(t, u)=\int_{R} \exp \{i u v\} \mathrm{d} \sigma_{t}(v)$, then the Fourier-Stieltzes transform has some properties that 1) for all $u \in R$, $|f(u)| \leq\left\|\sigma_{t}\right\|$ and $f(-u)=\bar{f}(u)$, where $\bar{z}$ denotes the conjugate complex of $z \in \mathbf{C}$. 2) $f$ is uniform continuous in $R$. To see this, we write for all $u$ and $h$,

$f(u+h)-f(u)=\int_{R}\left(\mathrm{e}^{i(u+h) v}-\mathrm{e}^{i u v}\right) \mathrm{d} \sigma_{t}(v)$ and $|f(u+h)-f(u)| \leq \int_{R}\left|\mathrm{e}^{i u v}\right|\left|\mathrm{e}^{i h v}-1\right| \mathrm{d}\left|\sigma_{t}\right|(v)$, where the last integrand is bounded 
by 2 and tends to 0 as $h \rightarrow 0$ for each $v \in R$ and the last integral is bounded by $2\left\|\sigma_{t}\right\|$. Hence the integral converges to 0 by the bounded convergence theorem. Since it does not involve $u \in R$, the convergence is uniform with respect to $u \in R$.

Notation. Let $\Delta_{n}(T)$ be defined by

$$
\Delta_{n}(T) \equiv\left\{\left(t_{1}, t_{2}, \cdots, t_{n}\right) \mid 0 \leq t_{1} \leq t_{2} \leq \cdots \leq t_{n} \leq T\right\}, t_{0}=0 .
$$

To expand the main result of this paper and to apply the Wiener integration formula and to prove the existence of the Wiener integral of $F(x)$ in (6), we need to express $\mathrm{F}(\mathrm{x})$ as the function of the form $f\left(x\left(t_{1}\right), x\left(t_{2}\right), \cdots, x\left(t_{n}\right)\right)$.

Lemma 3.2. Let $F: C_{0}[0, T] \rightarrow \mathbf{C}$ be defined by (6) and (7). Then we have that

$$
F(x)=\sum_{n=0}^{\infty} \int_{\Delta_{n}(T) \times R^{n}} \exp \left\{i \sum_{j=1}^{n} v_{j} \cdot x\left(t_{j}\right)\right\} \mathrm{d} \mu_{n}(\vec{t}, \vec{v})
$$

where $\mu_{n}$ is a countably additive Borel measure defined on $\Delta_{n}(T) \times R^{n}$ for each $n=1,2, \cdots, n$.

Proof. Using the series expansion of the exponential function, we have that

$$
\begin{aligned}
F(x)= & \exp \left\{\int_{0}^{T} \theta(t, x(t)) \mathrm{d} t\right\}=\sum_{n=0}^{\infty} \frac{1}{n !}\left[\int_{0}^{T} \theta(t, x(t)) \mathrm{d} t\right]^{n} \\
= & \sum_{n=0}^{\infty} \frac{1}{n !}\left[n ! \int_{\Delta_{n}(T)} \prod_{j=1}^{n} \theta\left(t_{j}, x\left(t_{j}\right)\right) \mathrm{d} \vec{t}\right] \\
= & \sum_{n=0}^{\infty} \int_{\Delta_{n}(T)} \theta\left(t_{1}, x\left(t_{1}\right)\right) \cdot \theta\left(t_{2}, x\left(t_{2}\right)\right) \cdots \theta\left(t_{n}, x\left(t_{n}\right)\right) \mathrm{d} t_{1} \mathrm{~d} t_{2} \cdots \mathrm{d} t_{n} \\
= & \sum_{n=0}^{\infty} \int_{\Delta_{n}(T)}\left[\int_{R} \exp \left\{i v_{1} x\left(t_{1}\right)\right\} \mathrm{d} \sigma_{t_{1}}\left(v_{1}\right)\right] \cdot\left[\int_{R} \exp \left\{i v_{2} x\left(t_{2}\right)\right\} \mathrm{d} \sigma_{t_{2}}\left(v_{2}\right)\right] \\
& \cdots\left[\int_{R} \exp \left\{i v_{n} x\left(t_{n}\right)\right\} \mathrm{d} \sigma_{t_{n}}\left(v_{n}\right)\right] \mathrm{d} t_{1} \mathrm{~d} t_{2} \cdots \mathrm{d} t_{n} \\
= & \sum_{n=0}^{\infty} \int_{\Delta_{n}(T)} \int_{R^{n}} \exp \left\{i \sum_{j=1}^{n} v_{j} \cdot x\left(t_{j}\right)\right\}\left[\prod_{j=1}^{n} \mathrm{~d} \sigma_{t_{j}}\left(v_{j}\right) \mathrm{d} t_{j}\right] \\
= & \sum_{n=0}^{\infty} \int_{\Delta_{n}(T) \times R^{n}} \exp \left\{i \sum_{j=1}^{n} v_{j} \cdot x\left(t_{j}\right)\right\} \mathrm{d} \mu_{n}(\vec{t}, \vec{v}),
\end{aligned}
$$

where $\mathrm{d} \mu_{n}(\boldsymbol{t}, \boldsymbol{v})=\left[\prod_{j=1}^{n} \mathrm{~d} \sigma_{t_{j}}\left(v_{j}\right) \mathrm{d} t_{j}\right]$ and $\sigma_{t_{j}} \in \mathbf{M}(R)$ is a complex Borel measure defined on $R$ and $\left\|\sigma_{t_{j}}\right\|<\infty$ for each $j=1,2, \cdots, n$ and $|F(x)| \leq \sum_{n=1}^{\infty}\left\|\mu_{n}\right\|<\infty$.

Remark. For more details about properties of the function $F(x)$ in (6) and (7), see the chapter 15 of the book [9]. Some properties of the exponential function of [9] give me a good motivation about this paper. Especially, the third equality in (10) follows from the Equation (15.3.17) in [9].

Theorem 3.3. For $z \in \mathbf{C}^{+}$and for each $j=1,2, \cdots, n$ and for functions $F: C_{0}[0, T] \rightarrow \mathbf{C}$ in (6) and for real $\rho>0$, the Wiener integral exists and is of the form: 


$$
\int_{C_{0}[0, T]} F(\rho x) \mathrm{d} m(x)=\sum_{n=0}^{\infty} \int_{\Delta_{n}(T) \times \mathbf{R}^{n}} \exp \left\{-\frac{\rho^{2}}{2} \sum_{j=1}^{n}\left(t_{j}-t_{j-1}\right) \sum_{k=j}^{n} v_{k}^{2}\right\} \mathrm{d} \mu_{n}(\boldsymbol{t}, \boldsymbol{v}),
$$

where $\mu_{n}$ is a countably additive complex Borel measure defined on $\Delta_{n}(T) \times R^{n}$ for each $n=1,2, \cdots$ and $\mathrm{d} \mu_{n}(\boldsymbol{t}, \boldsymbol{v})=\left[\prod_{j=1}^{n} \mathrm{~d} \sigma_{t_{j}}\left(v_{j}\right) \mathrm{d} t_{j}\right]$.

Proof. By the Wiener integration formula, we have that for real $\rho>0$,

$$
\begin{aligned}
\int_{C_{0}[0, T]} F(\rho x) \mathrm{d} m(x) & \\
= & \int_{C_{0}[0, T]}\left[\sum_{n=0}^{\infty} \int_{\Delta_{n}(T)}\left[\prod_{j=1}^{n}\left[\int_{R} \exp \left\{i \rho x\left(t_{j}\right) v_{j}\right\} \mathrm{d} \sigma_{t_{i}}\left(v_{j}\right)\right] \mathrm{d} \vec{t}\right]\right] \mathrm{d} m(x) \\
= & \prod_{j=1}^{n}\left(\frac{1}{2 \pi\left(t_{j}-t_{j-1}\right)}\right)^{\frac{1}{2}} \int_{R^{n}}\left[\sum_{n=0}^{\infty} \int_{\Delta_{n}(T)}\left[\prod_{j=1}^{n} \int_{R} \exp \left\{i \rho u_{j} v_{j}\right\} \mathrm{d} \sigma_{t_{j}}\left(v_{j}\right)\right] \mathrm{d} \vec{t}\right] \\
& \cdot \exp \left\{-\frac{1}{2} \sum_{j=1}^{n}\left[\frac{\left[u_{j}-u_{j-1}\right]^{2}}{t_{j}-t_{j-1}}\right\} \mathrm{d} \vec{u}\right. \\
= & \prod_{j=1}^{n}\left(\frac{1}{2 \pi\left(t_{j}-t_{j-1}\right)}\right)^{\frac{1}{2}} \int_{R^{n}} \exp \left\{-\frac{1}{2} \sum_{j=1}^{n} \frac{\left[u_{j}-u_{j-1}\right]^{2}}{t_{j}-t_{j-1}}\right\} \\
& \cdot\left[\sum_{n=0}^{\infty} \int_{\Delta(T) \times R^{n}} \exp \left\{i \rho \sum_{j=1}^{n} u_{j} v_{j}\right\}\left[\prod_{j=1}^{n} \mathrm{~d} \sigma_{t_{j}}\left(v_{j}\right) \mathrm{d} t_{j}\right]\right] \mathrm{d} \vec{u} \\
= & \sum_{n=0}^{\infty} \int_{\Delta(T) \times R^{n}}\left[\prod_{j=1}^{n}\left(\frac{1}{2 \pi\left(t_{j}-t_{j-1}\right)}\right)^{\frac{1}{2}} \int_{R^{n}} \exp \left\{-\frac{1}{2} \sum_{j=1}^{n} \frac{\left.u_{j}-u_{j-1}\right]_{j}}{t_{j}-t_{j-1}}\right\}\right. \\
& \left.\cdot \exp \left\{i \rho \sum_{j=1}^{n} u_{j} v_{j}\right\} \mathrm{d} \vec{u}\right] \mathrm{d} \mu_{n}(\vec{t}, \vec{v}) \\
= & \sum_{n=0}^{\infty} \int_{\Delta(T) \times R^{n}} \exp \left\{-\frac{\rho^{2}}{2} \sum_{j=1}^{n}\left(t_{j}-t_{j-1}\right) \sum_{k=j}^{n} v_{k}^{2}\right\} \mathrm{d} \mu_{n}(\vec{t}, \vec{v}),
\end{aligned}
$$

where $\mathrm{d} \mu_{n}(\boldsymbol{t}, \boldsymbol{v})=\left[\prod_{j=1}^{n} \mathrm{~d} \sigma_{t_{j}}\left(v_{j}\right) \mathrm{d} t_{j}\right]$. The last equality in (12) can be proved by the mathematical induction. $\square$

By the above result, we can investigate a very interesting behavior of the Wiener integral.

Definition 3.4. We define the scale factor for the Wiener integral by the varying real number $\rho>0$ such that

$$
G(\rho)=\left|\int_{C_{0}[0, T]} F(\rho x) \mathrm{d} m(x)\right|
$$

where $G: R \rightarrow C$ is a complex valued function defined on $R$.

Property 3.1. Behavior of the scale factor for the Wiener Integral.

We investigate the interesting behavior of the scale factor for the Wiener integral by analyzing the analytic Wiener integral as followings: For real $\rho>0$,

$$
\int_{C_{0}[0, T]} F(\rho x) \mathrm{d} m(x)=\sum_{n=0}^{\infty} \int_{\Delta_{n}(T) \times \mathbf{R}^{n}} \exp \left\{-\frac{\rho^{2}}{2} \sum_{j=1}^{n}\left(t_{j}-t_{j-1}\right) \sum_{k=j}^{n} v_{k}^{2}\right\} \mathrm{d} \mu_{n}(\vec{t}, \vec{v}) .
$$


Example 1. For the scale factor $\rho=\{\cdots, 1100,110,1,10,100, \cdots\}$, we can investigate the very interesting behavior of the Wiener integral:

1)

$$
\int_{C_{0}[0, T]} F\left(\frac{1}{100} x\right) \mathrm{d} m(x)
$$

$=\sum_{n=0}^{\infty} \int_{\Delta_{n}(T) \times \mathbf{R}^{n}} \exp \left\{-\frac{1}{2} \times 10^{4} \times \sum_{j=1}^{n}\left(t_{j}-t_{j-1}\right) \sum_{k=j}^{n} v_{k}^{2}\right\} \mathrm{d} \mu_{n}(\vec{t}, \vec{v})$

2)

$$
\int_{C_{0}[0, T]} F\left(\frac{1}{10} x\right) \mathrm{d} m(x)
$$

$$
=\sum_{n=0}^{\infty} \int_{\Delta_{n}(T) \times \mathbf{R}^{n}} \exp \left\{-\frac{1}{2} \times 10^{2} \times \sum_{j=1}^{n}\left(t_{j}-t_{j-1}\right) \sum_{k=j}^{n} v_{k}^{2}\right\} \mathrm{d} \mu_{n}(\vec{t}, \vec{v})
$$

3) $=\sum_{n=0}^{\infty} \int_{\Delta_{n}(T) \times \mathbf{R}^{n}} \exp \left\{-\frac{1}{2} \times 10^{0} \times \sum_{j=1}^{n}\left(t_{j}-t_{j-1}\right) \sum_{k=j}^{n} v_{k}^{2}\right\} \mathrm{d} \mu_{n}(\vec{t}, \vec{v})$ $\int_{C_{0}[0, T]} F(10 x) \mathrm{d} m(x)$

4) $=\sum_{n=0}^{\infty} \int_{\Delta_{n}(T) \times \mathbf{R}^{n}} \exp \left\{-\frac{1}{2} \times 10^{-2} \times \sum_{j=1}^{n}\left(t_{j}-t_{j-1}\right) \sum_{k=j}^{n} v_{k}^{2}\right\} \mathrm{d} \mu_{n}(\vec{t}, \vec{v})$

$$
\int_{C_{0}[0, T]} F(100 x) \mathrm{d} m(x)
$$

5) $=\sum_{n=0}^{\infty} \int_{\Delta_{n}(T) \times \mathbf{R}^{n}} \exp \left\{-\frac{1}{2} \times 10^{-4} \times \sum_{j=1}^{n}\left(t_{j}-t_{j-1}\right) \sum_{k=j}^{n} v_{k}^{2}\right\} \mathrm{d} \mu_{n}(\vec{t}, \vec{v})$

\section{Remark. <Interpretation of the scale factor for the Wiener integral>}

1) We can investigate the behavior of the Wiener integral as the varying scale factor by re-interpreting the analytic Wiener integral!

2) The exponential term of the Wiener integral is decreasing, whenever the scale factor $\rho>0$ is increasing. The exponential term of the Wiener integral is increasing, whenever the scale factor $\rho>0$ is decreasing.

3) The function $G: \rho \rightarrow\left|\int_{C_{0}[0, T]} F(\rho x) \mathrm{d} m(x)\right|$ is a decreasing function of $\rho>0$, because the exponential function $y=\mathrm{e}^{-x}$ is a decreasing function of $x \in R$.

That is, the absolute value of the Wiener integral is a decreasing function about the scale factor $\rho>0$ and

$$
\begin{aligned}
& \text { 1) } 0 \leq\left|\int_{C_{0}[0, T]} F(\rho x) \mathrm{d} m(x)\right| \leq \sum_{n=0}^{\infty}\left\|\mu_{n}\right\| \\
& \text { 2) } \lim _{\rho \rightarrow 0}\left|\int_{C_{0}[0, T]} F(\rho x) \mathrm{d} m(x)\right|=\sum_{n=0}^{\infty}\left\|\mu_{n}\right\| \\
& \text { 3) } \lim _{\rho \rightarrow+\infty}\left|\int_{C_{0}[0, T]} F(\rho x) \mathrm{d} m(x)\right|=0
\end{aligned}
$$

Conclusion. What we have done in this research is that we first define the 
scale factor for the Wiener integral and later, we investigate the very interesting behavior of the scale factor for the Wiener integral. From these results, we find a new property for the Wiener integral as a function of a scale factor!

Remark. The solution of the heat equation $\frac{\partial U}{\partial t}=-H U, U(0, \cdot)=\psi(\cdot)$ is

$$
U(t, \xi)=\left(\mathrm{e}^{-t H} \psi\right)(\xi)=E\left[\mathrm{e}^{-\int_{0}^{t} V(x(s)+\xi) \mathrm{d} s} \cdot \psi(x(t)+\xi)\right]
$$

where $\psi \in L_{2}\left(R^{d}\right)$ and $\xi \in R^{d}$ and $x(\cdot)$ is a $R^{d}$-valued continuous function defined on $[0, t]$ such that $x(0)=0$ and $E$ denotes the expectation with respect to the Wiener path starting at time $t=0$ and $H=-\Delta+V$ is the energy operator(or, Hamiltonian) and $\Delta$ is a Laplacian and $V: R^{d} \rightarrow R$ is a potential. This formula is called the Feynman-Kac formula. For more details, see the paper [8] and the book [9]

Remark. <Gratitude for the Refree> I am very gratitude for the referee to comment in details.

\section{Founding}

Research fund of this paper is supported by NRF-2017R1A6A3A11030667 as a research professor in the project of a National Research Foundation.

\section{References}

[1] Brue, M.D. (1972) A Functional Transform for Feynman Integrals Similar to Fourier Transform. PhD Thesis, University of Minnesota, Saint Paul.

[2] Cameron, R.H. (1954) The Translation Pathology of Wiener Space. Duke Mathematical Journal, 21, 623-628. https://doi.org/10.1215/S0012-7094-54-02165-1

[3] Cameron, R.H. and Martin, W.T. (1944) On Transformations of Wiener Integrals under Translations. Annals of Mathematics, 45, 386-396.

https://doi.org/10.2307/1969276

[4] Cameron, R.H. and Martin, W.T. (1945) Transformations for Wiener Integrals under a General Class of Linear Transformations. Transactions of the American Mathematical Society, 58, 184-219.

https://doi.org/10.1090/S0002-9947-1945-0013240-1

[5] Cameron, R.H. and Martin, W.T. (1947) The Behavior of Measure and Measurability under Change of Scale in Wiener Space. Bulletin of the American Mathematical Society, 53, 130-137. https://doi.org/10.1090/S0002-9904-1947-08762-0

[6] Cameron, R.H. and Storvick, D.A. (1987) Relationships between the Wiener Integral and the Analytic Feynman Integral. Supplemento ai Rendiconti del Circolo Matematico di Palermo, Serie II-numero, 17, 117-133.

[7] Cameron, R.H. and Storvick, D.A. (1987) Change of Scale Formulas for Wiener Integral. Supplemento ai Rendiconti del Circolo Matematico di Palermo, Serie II-numero, 17, 105-115.

[8] Gaysinsky, M.D. and Goldstein, M.S. (1992) Self-Adjointness of Schrödinger Operator and Wiener Integrals. Integral Equations and Operator Theory, 15, 973-990. https://doi.org/10.1007/BF01203123

[9] Johnson, G.W. and Lapidus, M.L. (2000) The Feynman Integral and Feynman's Operational Calculus. Oxford Science Publications, Oxford. 
[10] Johnson, G.W. and Skoug, D.L. (1979) Scale-Invariant Measurability in Wiener Space. Pacific Journal of Mathematics, 283, 157-176. https://doi.org/10.2140/pjm.1979.83.157

[11] Kim, Y.S. (1998) A Change of Scale Formula for Wiener Integrals of Cylinder Functions on the Abstract Wiener Space. International Journal of Mathematics and Mathematical Sciences, 21, 73-78. https://doi.org/10.1155/S0161171298000088

[12] Kim, Y.S. (2001) A Change of Scale Formula for Wiener Integrals of Cylinder Functions on Abstract Wiener Space II. International Journal of Mathematics and Mathematical Sciences, 25, 231-237. https://doi.org/10.1155/S0161171201004537

[13] Kim, Y.S. (2006) The Behavior of the First Variation under the Fourier Feynman Transform on Abstract Wiener Spaces. Journal of Fourier Analysis and Application, 12, 233-242. https://doi.org/10.1007/s00041-005-5050-5

[14] Kim, Y.S. (2010) Fourier Feynman Transform and Analytic Feynman Integrals and Convolutions of a Fourier Transform of a Measure on Wiener Spaces. Houston Journal of Mathematics, 36, 1139-1158.

[15] Kim, Y.S. (2010) Behavior of the First Variation under a Fourier-Feynman Transform for Cylinder Functions on Wiener Spaces II. Integral Transforms and Special Functions, 21, 13-23. https://doi.org/10.1080/10652460902945100

[16] Kim, Y.S. (2016) Behavior of the First Variation of Fourier Transform of a Measure on the Fourier Feynman Transform and Convolution. Numerical Functional Analysis and Optimization, 37, 699-718. https://doi.org/10.1080/01630563.2016.1173700 